\title{
Algorithm for solving the inverse problem of pharmacokinetics to determine the transition coefficients
}

\author{
A. Takuadina \\ L.N. Gumilev Eurasian National University, Astana, Kazakhstan \\ e-mail:alyoka.01@mail.ru
}

Key words: mathematical model, optimization method, inverse problems of pharmacokinetics

\begin{abstract}
Motivation and Aim: In this article, a mathematical model of blood circulation of the human body is considered, representing both a set of chambers. By taking blood from the patient, the concentration of the drug in the first chamber is determined, which we take as additional information for solving the inverse problem. According to the known additional information, it is necessary to determine all transition coefficients (rate constants).
\end{abstract}

Methods and Algorithms: To solve the inverse problem, we use the optimization method. The essence of this is to minimize the quadratic residual functional of the observed and calculated state of the drug concentration in the chamber. As the formulation of a direct problem, a system of kinetic equations is used that describes the movement of the preparation from the chamber to the chamber.

Results: An auxiliary (adjoint) problem is constructed, with the help of which an explicit form of the gradient of the minimized functional is obtained. A difference analogue of the algorithms for solving the direct and conjugate problem formulated in the discrete formulation [1] is developed.

Acknowledgements: to the foreign scientific adviser: Corresponding Member of the Russian Academy of Sciences, Director of the Institute of Computational Mathematics and Mathematical Geophysics, Doctor of ph.-m. sciences, Professor Kabanikhin Sergey Igorevich.

\section{References}

1. Kabanikhin S.I. (2009) Inverse and ill-posed problems. Novosibirsk: Siberian Scientific Publishing House. 457 p. 